\title{
NQS - a graphical query system for data models with binary relationship types
}

\author{
H.-J. Klein ${ }^{1}$, D. Krämer ${ }^{2}$ \\ ${ }^{1}$ Institut für Informatik und Praktische Mathematik \\ ${ }^{2}$ Rechenzentrum der Universität \\ Universität Kiel, Olshausenstr. 40, 24098 Kiel, Germany
}

\begin{abstract}
A graphical query system for data models with binary relationship types is described in which queries can be formulated as annotated graphs. The concepts of the underlying descriptive query language allow to represent even complex queries in a graphical form that is easy to understand. Identifiers with local scope and the possibility to build queries in a step by step manner help to avoid problems sometimes mentioned in connection with the formulation of complex queries in descriptive query languages. Query formulation is supported by comfortable operations on graphs and by an efficient layout algorithm.
\end{abstract}

Keywords

Entity-Relationship data model, graphical user interface, visual query language, query graphs, schema layout algorithm

\section{INTRODUCTION}

There are numerous proposals in the literature for query interfaces applying graphics with the intention to make the interactive access to databases more comfortable for the expert and less difficult for the casual user. Among the first such proposals are QbE (Zloof (1975)), CUPID (Mc Donald (1975)), and TIMBER (Stonebraker (1982)) for the relational data model. Most graphical query systems, however, have been developed for data models which allow to model relationships between objects explicitly by named relationship types. The explicit representation of relationship types allows to visualize schemas in diagrammatic form such that complex schemas are better to understand than relational schemas where relationships are modelled by identical values in attributes having the same domain. For the basic Entity-Relationship (ER) data model and extensions of the model, graphical query systems have been described for example in Wong (1982), Zhang (1983), Elmasri (1985), Burns (1988), Kuntz (1989), and Angelaccio (1990); proposals for other data models can be found in King (1984), Mark (1989), Lam (1990), and ACM (1992). 
The term 'graphical' is used for a wide spectrum of query interfaces with quite different characteristics. On one side of this spectrum there are 'procedural' facilities which allow to browse through databases at the level of schema/state diagrams by typing in commands or by using the mouse (e.g.: Burns (1988), Rogers' (1988)). On the other side there are 'descriptive' query systems and proposals of 'descriptive' query languages which allow to specify queries more or less completely as annotated graphs derived from schema diagrams (e.g.: Bryce (1986), Kim (1988), Czejdo (1990), Lam (1990), Mohan (1993)). Grouped between these two classes of query interfaces there are facilities where queries are formulated as a combination of some portion of the schema diagram (eventually with modifications) and text at different locations of the screen (e.g.: Wong (1982), Burns (1988)). Further remarks on the use of the term 'graphical' in connection with query interfaces can be found in Kuntz (1989).

For many graphical query interfaces, there is no underlying query language given; thus, query semantics has to be defined procedurally based on the graphical operations of the interface. In NQS the result of each graphical query specification accepted by the system is an annotated query graph which corresponds uniquely (up to equivalence) to a textual query of the query language NQL (Network Query Language, Klein (1989)). Since semantics of NQL is formally defined, formal semantics is provided for the interface, too. The reference to a textual query language does not mean that the graphical specification of a query in NQS is guided by its textual counterpart (as, for example, in Lam (1990)) or that the user has to memorize the exact syntax of NQL. Query graphs may rather be constructed under guidance of NQS in any reasonable order.

NQL has been proposed as a high level descriptive query language for the CODASYL data model. However, the language can be used as query language for all data models with simple object types and binary relationship types because it does not refer to special features of the CODASYL data model such as the restriction of binary relationships to 1 to $n$ relationships or the ordering of members in (CODASYL) sets.

In descriptive languages, queries are formulated with the help of predicates on the level of database schemas. This kind of query formulation may be characterized as 'logical' navigation in order to distinguish it from navigation on the level of objects in procedural languages. Predicates on the level of database schemas must allow to address all kinds of relationships between objects. For data models with explicit relationship types this means that predicate forms have to be available for explicit relationships as well as for value-based relationships and combinations of both kinds of relationships.

There are a number of descriptive languages proposed for the ER data model and other data models with explicit relationship types (e.g.: Deheneffe (1976), Markowitz (1983), Subieta (1987), Mohan (1993)). The main difference between NQL and these proposals is the form in which language constructs are adapted to the idea of logical navigation. Complex relationships between objects can be addressed in NQL by Boolean expressions over paths with relationship types and object types as elements. There are no variables referencing objects or values of objects in the language but the identifiers of object types are used with local scope instead. This use of identifiers conforms well to the idea of logical navigation. Predicate forms provided for the different kinds of relationships allow to express quantification in such a way that queries often look like English sentences (there is some similarity in this regard with ILL (Lacroix (1977)), a domain-oriented query 
language for the relational data model). Complex queries can be formulated in a series of steps with seperation of the specification of the target list from parts where quantifiers are needed.

The concepts underlying NQL make this language a suitable candidate as reference language for a query interface with queries represented completely as graphs and with emphasis on both the readability of query specifications and the ease of interpretation. The features of this interface help to avoid problems with the formulation of complex queries in descriptive languages reported in Welty (1981) and Hansen (1987).

A query graph is generated in NQS by modification of a diagram which is automatically produced from the textual description of a given schema. Among the possible modifications are the addition of new nodes and edges as well as the deletion of existing ones. In order not to upset the user by layouts changing drastically, NQS keeps the relative positioning of object types in the initial diagram during all stages of query formulation.

The rest of the paper is organized as follows. In the next section, a brief description of the visualization of schemas and query graphs in NQS is given. The third section demonstrates with several examples how queries are specified in NQS and how query graphs have to be interpreted. Some comments on experiences with NQS follow. The final section contains some technical details of the system and describes future directions of its development.

\section{Drawing of Schema Diagrams and Query Graphs}

The specification of queries as annotated graphs derived from schema diagrams should be fully supported by the query system. This especially means that a user has not to deal with the placement or rearrangement of graphical objects. A second demand is that during query formulation the layout of schema components must not change drastically, i.e. familiarity with the visual representation of the schema (relative positioning) has to be preserved. A third feature is the readability of query graphs. The number of different types of graphical symbols should not be too large. Last but not least efficiency has to be guaranteed during all steps of query formulation.

There are a number of layout algorithms for schema diagrams (see Tamassia (1988), Paulisch (1993)). In NQS we make use of the efficient embedding algorithm for planar graphs given in Chiba (1985). This algorithm is based on an algorithm of Booth (1976) which improved the planarity testing algorithm of Lempel (1967) by introducing a data structure called a PQ-tree and by employing an efficient st-numbering algorithm (Even (1976)). It is easy to implement and the PQ-tree data structure has the nice property to allow a simple realization of high-level functions for the generation of different embeddings. Thus, the algorithm is very attractive for the interactive manipulation of schema layouts. Such a layout manipulation under user control may become advantageous in NQS especially after the insertion of new schema elements.

Graph algorithms can be applied to schema diagrams for data models with binary relationship types in a straightforward manner by identifying object types with the nodes of a graph and relationship types with its edges. Schema diagrams considered as graphs in this way are often seperable graphs with many biconnected blocks (e.g.: the diagram in 
Figure 1 has 5 blocks). The algorithm of Chiba (1985) supposes graphs to be nonseperable. However, it is not difficult to extend this algorithm such that it can be applied in a single step to seperable graphs without loss of efficiency (linear time complexity).

Schema diagrams are also often nonplanar. When running into situations which are in conflict with planarity, an appropriate alteration of the PQ-tree data structure allows to continue with the algorithm such that it is possible to find embeddings of nonplanar graphs, too. The result of the embedding algorithm modified so far is an ordered adjacency list for each node together with an extended st-numbering and information on the connectedness of the graph.

Given this result, different algorithms can be used to generate an appropriate drawing of the schema diagram. What has to be considered is that nodes of a schema diagram may be quite different in size if information like attributes or conditions is shown within the node and not hidden in pop-up's. In NQS, a simple algorithm produces the diagram in a tree-like manner with depth-first recursion controlled by the extended st-numbering. If there are crossing edges because of nonplanarity, they are drawn in such a way that no misleading layout results.

Our layout algorithm takes as input the textual description of a complete CODASYL database schema or of an ER schema which can be generated interactively. Figure 1 presents a schema diagram drawn by this algorithm. The algorithm can also be applied to draw query graphs in a way which preserves visual familiarity with the schema diagram: the drawing of a query graph is done in the same manner as the drawing of the schema diagram with missing nodes having no size, i.e. consuming no place. Additional nodes produced by duplication of existing nodes or by the insertion of user-defined object types (see below) are placed next to their source node.

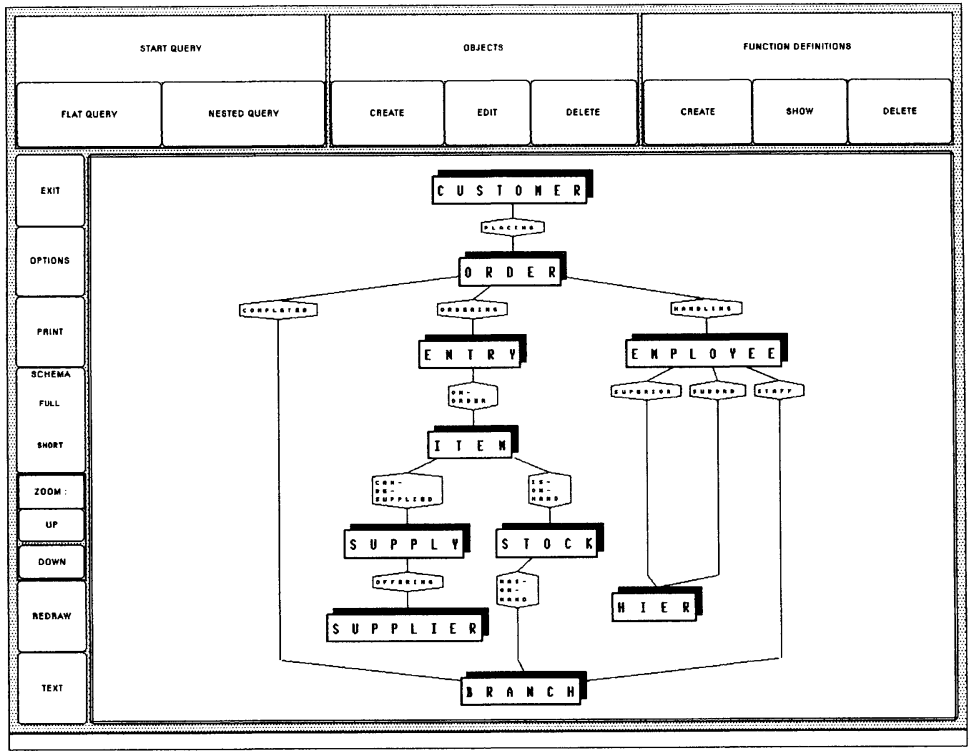

Figure 1 NQS interface with a schema diagram drawn by the embedding algorithm 


\section{QUERY Formulation}

Query formulation in NQS means transformation of a given schema diagram into a query graph by use of graphical functions and by entering some text, if necessary. A graph is accepted by the system as a correct query graph if it represents a query of the textual query language NQL. Query graphs are also used for the specification of user-defined object types and for the definition of function expressions. The transformation of query graphs into NQL queries can be formally specified in a straightforward manner with the help of functions which map nodes and edges to the corresponding textual terms.

As in schema diagrams, nodes in query graphs represent object types or relationship types. Nodes representing object types, however, are further distinguished whether they represent so-called selected elements or path elements. Selected elements of a query are those object types which are addressed in the target list of the query, whose attribute values are restricted by conditions, and/or which are quantified. All other object types are called path elements since they are only necessary for the specification of paths between selected elements. In order to characterize their role graphically, nodes representing path elements are always drawn by NQS without attributes and smaller in size than nodes representing selected elements.

Query formulation may start with the diagram of an original schema, i.e. a diagram which has been generated from the textual description of a schema, or it may start with the diagram of a modified version of such a schema. Schemas can be modified by the addition of user-defined object types and their relationship types (see below). At first, the user specifies that portion of the diagram which is relevant for the intended querying. This specification is done in two steps. In the first step, object types have to be marked as selected elements by pointing with the mouse at their representation. When selecting these elements a user can take advantage of the following completion method applied by NQS: for each pair of selected elements, NQS adds the shortest path connecting them in the schema diagram; if there are several shortest paths for such a pair, all these paths are added. A path can be given by a single relationship type or by an alternating sequence of relationship types and object types.

This completion method is of a purely syntactic nature and has always a unique result. For data models with different kinds of relationship types other completion methods could be of interest (Ioannidis (1994)). As a result of this first step, NQS generates a diagram with all nodes which have been marked shown as selected elements and with all other nodes shown as path elements.

In the second step of the specification, the user must delete those paths which are not addressed in the query. If additional paths are needed or if elements not selected in the first step shall be added, this can be done in the ADD-mode where the system presents the initial diagram again. Sometimes nodes must be marked in order to add paths needed in the query and not for the purpose of determining them as selected elements. Therefore it is possible in a further mode to change selected elements into path elements and vice versa.

As a consequence of this proceeding, it is always guaranteed that there are no incomplete paths in the diagram. Paths not shown in the diagram, on the other hand, have nothing to do with the meaning of the query. What has to be taken into account in these 
steps is that in the final query graph all path elements must have exactly two neighbours. This is a necessary condition for paths to represent exactly one (complex) relationship type between two selected elements.

\subsection{Flat queries}

Flat NQL queries allow to express simple conditions on attribute values and relationships between objects of those types which are used in the target list of the query. In simple cases, conditions for relationships result immediately from the paths in the query graph taken over from the schema diagram: objects of the database state qualify for the answer if they are connected in accordance with the paths in the query graph. Thus, the result of this type of query corresponds to a set of (not necessarily connected) subgraphs from the database state if states are considered as graphs with objects as nodes and relationships as edges.

The graphical representation of flat queries in NQS shows simple comparison expressions for attribute values as part of the graphical representation of selected elements and explicit relationships which have to be present between qualifying objects as paths. In Figure 2, objects of the two selected object types ORDER and BRANCH qualify for the answer if their attribute values are as specified and if they are connected in accordance with the path between them in the graph.

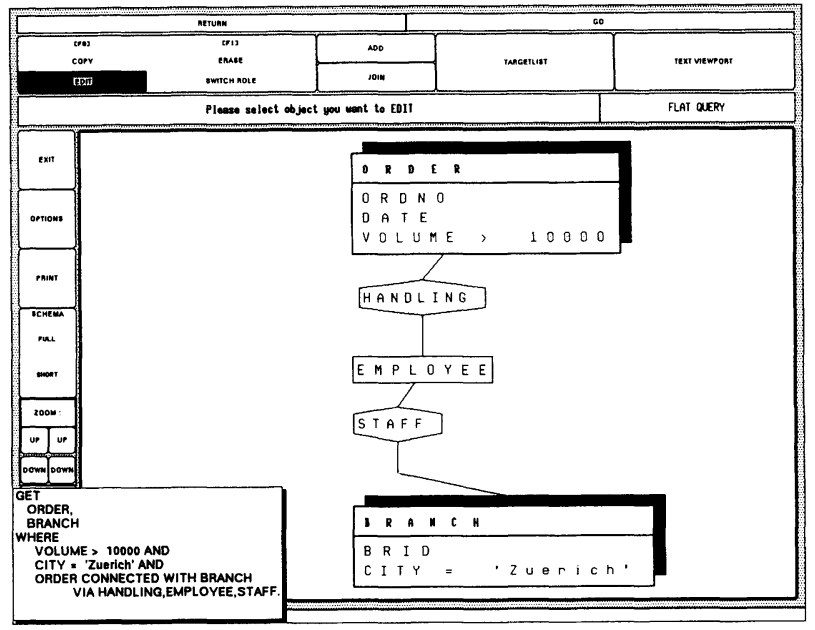

Figure 2 Get the data of each branch in Zuerich handling orders with volume greater than 10000 together with the data of these orders.

In flat queries it is also possible to compare values of objects of different type. To insert such a condition into the query graph, a user marks two attributes of different object types and chooses a comparison operator. The system draws an edge between the attributes and labels the edge with the comparison operator. Figure 3 shows a query graph for a flat query with a simple path and with both forms of comparison expressions. 

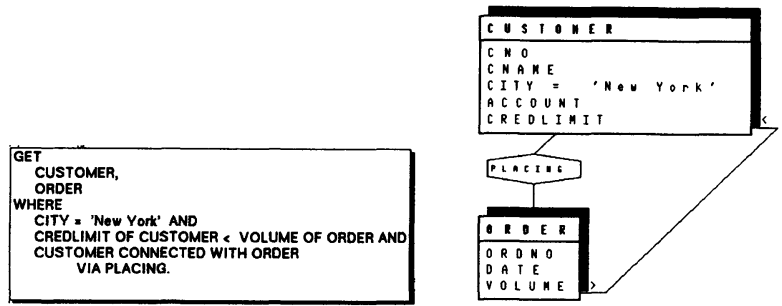

Figure 3 Get the data of all customers from New York who have placed orders with volume greater than their credit limit together with the data of these orders.

Predicates represented by paths in query graphs are connected by the conjunctive operator AND to predicates specified by comparison expressions in selected elements. This is a natural way to interpret query graphs. If predicates shall be connected otherwise, a corresponding Boolean expression has to be specified textually in the so-called text viewport. An expression in this viewport is connected by AND to the expression represented by the query graph itself. To make textual input short and comfortable, the system allows to mark paths automatically and to use the generated marks in Boolean expressions.

For flat queries, textual specification of Boolean (and arithmetic) expressions in a seperated viewport has been prefered to graphical representation for two reasons. The first reason is that the graphical representation of Boolean expressions tends to overload the query graph. The second reason is that in general there is no distinguished location where to pin a Boolean (or arithmetic) expression in the query graph (for nested queries there is always such a location).

As shown in Figures 2 and 3, a user can ask the system for the textual formulation corresponding to a graphical specification. This facility proved to be very helpful not only for the novice user but also for the expert to check whether the query graph represents the meaning of the intended query.

\subsection{Nested queries}

Semantics of flat NQL queries can be specified by quantifier-free formulas if there are predicate forms not only for comparison expressions but also for paths (Klein (1989)). More complex queries can be formulated in NQL by nesting quantified subexpressions to arbitrary depth. Compared with calculus-like languages, e.g. the relational tuple calculus, there is a different concept with respect to variables. Object types are not referenced by variables but their identifiers are used instead exclusively. The scope of these identifiers is restricted to the upper level of the where-clause following it in the query. Besides SOME and EVERY (ALL) there are further 'quantifiers' like NO, ONLY, AT LEAST n, etc. in the language. Quantifiers are attached directly to identifiers. This leads to more natural language like formulations. The target list of a nested query refers to exactly one object type. The idea here is to split complex queries into a sequence of definitions determining named subsets of object sets of the database state (cf. the 'data-context' in NUL (Deheneffe (1976))) and a final flat query in which these subsets can be referenced like ordinary object types. 
The local scope of identifiers and the possibility to build queries in a step by step fashion help to overcome 'linguistic complexity problems' mentioned in Hansen (1987) in connection with the formulation of complex queries in descriptive query languages. As in procedural languages, it is possible to interpret parts of query specifications without having to grasp the complete specification.

Let us first consider nested queries without predicates for value-based relationships. Because of the restriction that only a single object type can be referenced in the targetlist of a nested query and because of the restricted scope of identifiers, such a query can always be represented by a query graph with the following properties: there is one distinguished node, the target node; there are no cycles in the graph apart from those cycles which exist between two selected elements because of distinct paths connecting them; if such paths exist, they do not have common elements and they do not have selected elements as members; with the exception of these cycles, the graph is a tree with the target node as root.

Because of this tree property, all paths in a nested query graph have a unique direction into which they have to be read. Thus, there is no ambiguity with respect to the interpretation of quantification.

Target nodes in nested query graphs are marked by placing GET in front of the identifier. The graph in Figure 4 shows two selected elements, ORDER and BRANCH, which are connected by two paths. For each qualifying object of type ORDER, the

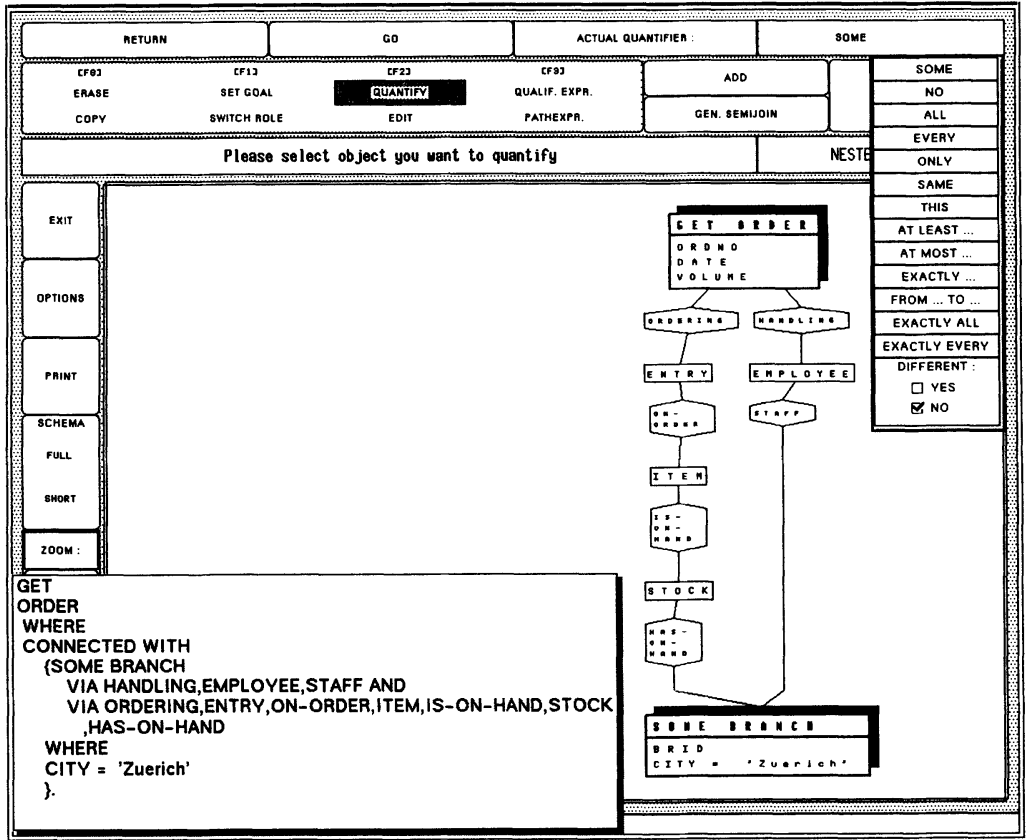

Figure 4 Get the data of all orders handled by some employee of a branch in Zuerich such that an item is ordered in the order which is on hand in this branch. 
following must hold: there is an object of type BRANCH with 'Zuerich' as value in the attribute CITY such that the ORDER object is connected with this BRANCH object as specified by the paths in the graph.

If two or more paths shall represent alternative connections between objects or if there are more complicated conditions on paths, a Boolean expression can be specified with path marks as operands. The marks are generated automatically by the system. The expression is placed into an extra field in the representation of the object type to which the paths are directed.

If there are object types which are addressed more than once in a query, these object types must be replicated in an appropriate number as nodes in the query graph. This corresponds to the independence of different occurrences of object identifiers in a nested textual query. Duplication of nodes is done by use of a function key. Figure 5 gives an example for this concept.
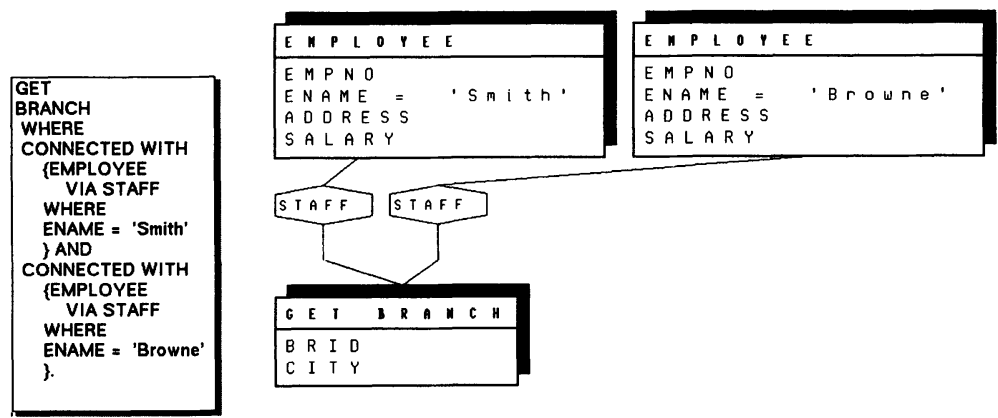

Figure 5 Get the data of all branches with an employee named Smith as well as with an employee named Browne on the staff.

If no quantifier is specified as for the two occurrences of the object type EMPLOYEE in the query of Figure 5, then SOME is implicit.

To refer back to an object type in a query, the keyword SAME (or THIS) can be used like an ordinary quantifier. The natural restriction is that the occurrence of an object type with this keyword is a leaf in the query tree.

Predicates for value-based relationships are called generalized semijoins since in these predicates all quantifiers and comparison operators can be used. They are represented in a query graph as follows: the representation of the object type for which the predicate must hold is extended by an extra field containing the comparison expression of the predicate. Each operand whose attributes originate in another object type is connected to the representation of that object type. Figure 6 shows an example.

In the query of Figure 6 there is no condition given concerning paths via explicit relationships between objects of type CUSTOMER and BRANCH. The next example demonstrates how such conditions can be specified by simply combining the corresponding edge with a path. If there are several paths involved, they are drawn seperately. Boolean expressions are handled as described above. Since simple edges and edges combined with 

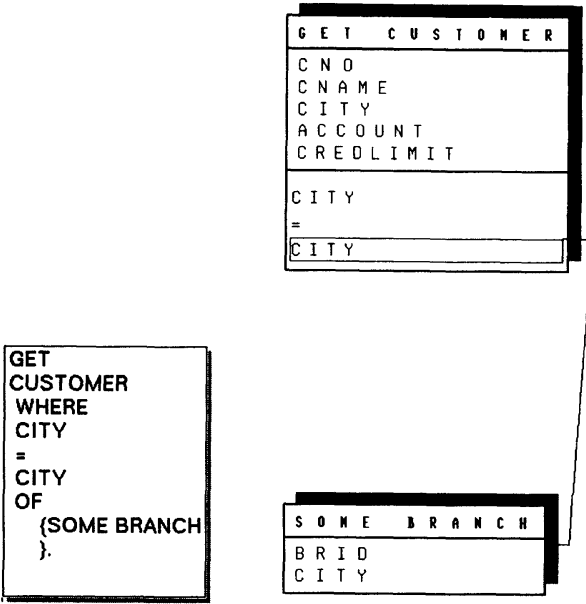

Figure 6 Get the data of all customers who live in a city where some branch is located. a path in the representation of a generalized semijoin predicate have a unique direction, the property that all paths in query graphs are directed away from the target node is preserved.
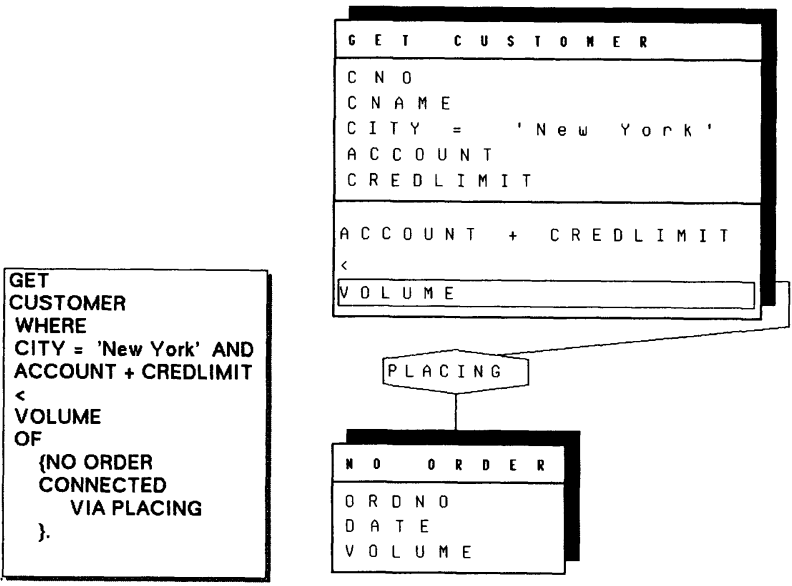

Figure 7 Get the data of all customers from New York who have placed no order with volume greater than the sum of their account and their credit limit.

Both operands in the comparison expression of a generalized semijoin predicate may refer to object types which are different from the object type the predicate belongs to. An example is given in Figure 8. 


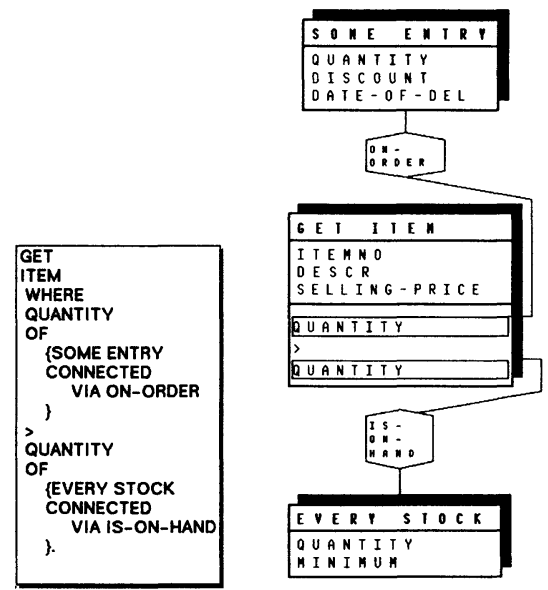

Figure 8 Get the data of all items with an entry in which the item is ordered with quantity greater than the quantity which is on hand of the item in each stock.

\subsection{User-defined object types and function expressions}

For each object type of the schema under consideration it is possible to specify an arbitrary number of nested queries and to give a unique identifier to each of them. After the completion of such a definition, the schema diagram is extended by the representation of the new object type with the identifier just introduced. This object type inherits all attributes and relationship types from the object type to which the nested query refers. It is drawn next to it and can be used for query specification or further definitions of new object types in the same way as any object type of the original schema. The specification of a new object type can be edited at any time. Updates producing cycles in a set of definitions are rejected by the system.

With the help of such definitions, a user can decompose query formulation into several steps and produce a special view of a database conforming to his/her needs. Figure 9 shows a query with user-defined object types.

The query graph of Figure 9 contains a simple Boolean expression as part of an additional field in the representation of the object type ITEM. In nested queries, Boolean expressions are uniquely attached to object types. Therefore NQS offers no viewport for Boolean expressions as in the case of flat queries. Conditions which can be used in a Boolean expression for an object type are marked by the system on request.

There are two types of function expressions in NQL called free and context-related. The current implementation of NQS restricts the use of function expressions to free function expressions. The specification of these expressions is done in a way similar to the definition of new object types. The only difference is that an aggregate function has to be specified which is applied either to an object set (NUMBER OF) or to values of arithmetic expressions with operands taken from the 'root' object type (SUM, AVG, ...). The identifiers of function expressions can be used like constants in queries. 

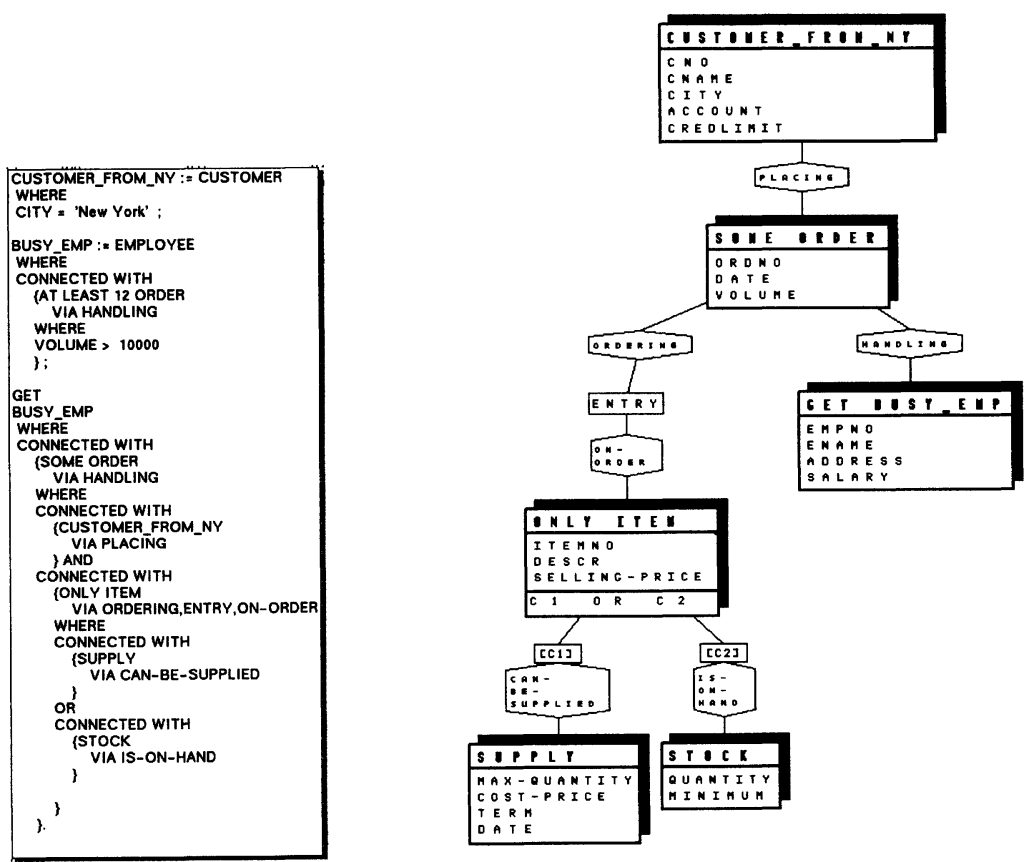

Figure 9 Get the data of all busy employees who work on some order of a New York customer requesting in this order only items which can be supplied or which are on hand.

\section{EXPERIENCES}

In the design phase of NQS we experimented with graphical representations for Boolean expressions in order to get an interface with a minimum of text entry. However, since the query language allows comparison expressions and predicates involving paths to be combined in Boolean expressions, graphical representations for more complex expressions often showed to be in conflict with the readability of query graphs. In order to keep the number of different concepts small, we therefore decided in favour of the textual specification of all Boolean expressions which are not simple conjunctions.

Our experience with the usability of NQS results from a large number of sessions where we gathered qualitative data from user comments and user performance. Several users, among them colleagues, students who had completed a course on database systems, and programmers with no database experience, took part in a test where some flat and nested. queries had to be formulated with help restricted to the interaction with the system. These users were not familiar with the query language NQL.

The first step in query formulation with NQS is to decide whether a given query can be specified as a flat query or as a nested query (or both). To come to a decision on the basis of the given formulation was not difficult for most users. The specification of flat queries also proved not to be a hard problem. Some argumentation, however, was 
necessary with regard to our decision for a text viewport since there were test queries for which a complete graphical representation is attractive.

Difficulties with nested queries showed to be strongly dependent upon the given formulation of a query in natural language. Our observations in this point were the following: if a given query formulation had a nested structure free from complex self-references of objects, then query specification was done in a step by step manner without problems. Quantification in particular was not considered a difficult concept. In case of more complicated formulations several attempts were sometimes necessary to find the nested structure of a correct specification. As an example consider the query in Figure 4: 'Get the data of all orders handled by some employee of a branch in Zuerich such that an item is ordered in the order which is on hand in this branch.' Figure 4 shows a simple graph for this query: take ORDER and BRANCH as selected elements and connect these elements via ITEM and EMPLOYEE, resp.; add the appropriate selection condition to BRANCH. Most users, however, tried to specify the query by duplicating the object types BRANCH or ORDER and by using SAME (THIS) as 'quantification' (there is a solution in this way by duplication of ORDER).

In order to check whether the generated query graph represented what they intended to specify, users always looked at the textual query formulation produced by NQS on demand. They had no difficulties to understand these NQL queries in connection with the query graph and to compare them with the given formulation of the query in natural language. As a consequence of this control and because NQS accepts only query graphs which correspond to syntactically correct NQL queries, final solutions were without mistakes in most cases.

\section{Final REMARKS}

NQS has been implemented in Pascal on Apollo workstations using Domain Dialogue and GMR2D. At each stage of query formulation, the user may ask the system for information about the graphical objects on the screen (e.g.: data types of attributes, specification of user defined object types). For the specification of the target list, arithmetic expressions, predicates etc., pop-up windows and pull-down menues are provided. In the current version, not all features of NQL are realized, among them the transitive closure of path expressions and context-related function expressions. The size of the system without interpreters for NQL and with a restricted facility for the presentation of query results is about 32000 lines of code (1.4 MB).

Not every diagram produced by the layout algorithm of NQS will be to the pleasure of the user. Moreover, the building of views by the specification and insertion of object definitions and the duplication of schema elements in query graphs may result in unfavourable layouts. Therefore we work on a facility which offers operations at different levels to permit the change of layouts. On a higher level, there shall be operations based on the information about the connectedness of the graph including those operations given in Chiba (1985) for finding all the embeddings of a planar graph (permutation and flip over of components); on a lower level, some simple rearrangement operations may be allowed. This proceeding seems to be more promising than to permit the user only to drag 
graphical objects arbitrarily over the screen.

NQL is not a complete language in the sense of a full calculus language because of the restrictions concerning the scope of identifiers and the form of target lists. In many query languages with descriptive features, completeness is achieved by set operations, i.e. procedural constructs (e.g.: Czejdo (1990), Angelaccio (1990)). For adding expressive power, we intend to go another way and to generalize the restricted view concept of the language such that complex object types can be specified by an extended form of nested queries.

Currently, a new version of NQS is under construction using an X11 based graphical toolkit. NQS is developed into a query interface for an extended version of the ER data model comprising subtypes, aggregation, and attributes for relationship types.

\section{REFERENCES}

ACM (1992) Special Issue: Advanced User Interfaces for Database Systems. ACM SIGMOD RECORD, 21 (1)

Angelaccio M., Catarci T., and Santucci G. (1990) QBD* : A Graphical Query Language with Recursion. IEEE Trans. on Software Engineering, 16 (10), 1150-1163

Booth K.S. and Lueker G.S. (1976) Testing the Consecutive Ones Property, Interval Graphs, and Planarity Using PQ-Tree Algorithms. Journal of Computer and System Sciences, 13, 335-379

Bryce D. and Hull R. (1986) SNAP: A Graphics-based Schema Manager, in Proc. IEEE Int. Conf. on Data Eng., Los Angeles, 151-164

Burns L.M., Archibald J.L., and Malhotra A. (1988) A Graphical Entity-Relationship Database Browser, in Proc. 21st Annual Hawaii Int. Conf. on System Sciences, Software Track vol. II, 694-704

Chiba N., Nishizeki T., Abe S., and Ozawa T. (1985) A Linear Algorithm for Embedding Planar Graphs Using PQ-Trees. Journal of Computer and System Sciences, 30, 54-76

Czejdo B., Elmasri R., Rusinkiewicz M., and Embley D.W. (1990) A Graphical Data Manipulation Language for an Extended Entity-Relationship Model. IEEE Computer, 23, $26-36$

Deheneffe C. and Hennebert H. (1976) NUL: a Navigational User's Language for a Network Structured Data Base, in Proc. ACM SIGMOD, Washington, 135-142

Elmasri R.A. and Larson J.A. (1985) A Graphical Query Facility for ER Databases, in Proc. 4th Int. Conf. on the Entity-Relationship Approach, Chicago, 236-245

Even S. and Tarjan R.E. (1976) Computing an st-numbering. Theoretical Computer Science, 2, 339-344

Hansen G.W. and Hansen J.V. (1987) Procedural and Non-procedural Query Languages Revisited: a Comparison of Relational Algebra and Relational Calculus. International Journal of Man-Machine Studies, 26, 683-694

Ioannidis Y.E. and Lashkari Y. (1994) Incomplete Path Expressions and their Disambiguation. ACM.SIGMOD RECORD, 23 (2), 138-149

Kim H.-J., Korth H.F., and Silberschatz A. (1988) PICASSO: A Graphical Query Language. Software-Practice and Experience, 18 (3), 169-203 
King R. and Melville S. (1984) Ski: A Semantics-Knowledgeable Interface, in Proc. 10th $V L D B$, Singapore, $30-33$

Klein H.-J. (1989) Pragmatics and Semantics of NQL, a Descriptive Query Language for Network Databases. Information Systems, 14 (1), 29-45

Kuntz M. and Melchert R. (1989) PASTA-3's Graphical Query Language: Direct Manipulation, Cooperative Queries, Full Expressive Power, in Proc. 15th VLDB, Amsterdam, 97-105

Lacroix M. and Pirotte A. (1977) Domain-oriented Relational Languages, in Proc. 3rd $V L D B$, Tokyo, 370-378

Lam H., Chen H.M., Ty F.S., Qiu J., and Su S.Y.W. (1990) A Graphical Interface for an Object-Oriented Query Language, in Proc. 14th IEEE COMPSAC, Chicago, 231-237

Lempel A., Even S., and Cederbaum I. (1967) An Algorithm for Planarity Testing of Graphs, in Theory of Graphs (ed. P. Rosenstiel), Gordon\&Breach, New York, 215-232,

Mark L. (1989) A Graphical Query Language for the Binary Relationship Model. Information Systems, 14 (3), 231-246

Markowitz V.M. and Raz Y. (1983) ERROL: an Entity-Relationship Role Oriented Query Language, in Entity-Relationship Approach to Software Engineering (P.A. Ng et al., eds.), North-Holland, 329-346

Mc Donald N. and Stonebraker M. (1975) CUPID - The Friendly Query Language, in Proc. ACM Pacific 75 Conf., San Francisco, 127-131

Mohan L. and Kashyap R.L. (1993) A Visual Query Language for Graphical Interaction With Schema-Intensive Databases. IEEE Trans. on Knowledge and Data Engineering, 5 (5), 843-858

Paulisch F.N. (1993) The Design of an Extendible Graph Editor. Lecture Notes in Computer Science, 704, Springer Verlag

Rogers T.R. and Cattell R.G.G. (1988) Entity-Relationship Database User Interfaces, in Proc. 6th Int. Conf. Entity-Relationship Approach (ed. S.T. March), New York, Elsevier, 353-365

Stonebraker M. and Kalash J. (1982) TIMBER: A Sophisticated Relation Browser, in Proc. 8th VLDB, Mexico City, 1-10

Subieta K. (1987) Denotational Semantics of Query Languages. Information Systems, 12 (1), 69-82

Tamassia R., Di Battista G., and Batini C. (1988) Automatic Graph Drawings and Readability of Diagrams. IEEE Trans. on Systems, Man, and Cybernetics, 18 (1), 61-79

Tarjan R. (1972) Depth-first Search and Linear Graph Algorithms. SIAM Journal of Computing, 1 (2), 146-160

Welty C. and Stemple D.W. (1981) Human Factors Comparison of a Procedural and a Nonprocedural Query Language. ACM Trans. on Database Systems, 6 (4), 626-649

Wong H.K.T. and Kuo I. (1982) GUIDE: Graphical User Interface for Database Exploration, in Proc. 8th VLDB, Mexico City, 22-32

Zhang Z.-Q. and Mendelzon A.O. (1983) A Graphical Query Language for EntityRelationship Databases, in Entity-Relationship Approach to Software Engineering (C.G. Davis et al., eds.), 441-448

Zloof M.M. (1975) Query by Example, in AFIPS Conf. Proc., National Computer Conf. 44, Anaheim, 431-438 


\section{BIOGRAPHY}

Hans-Joachim Klein is a member of the database group in the computer science department of the university of Kiel. He received the Diploma in computer science from the university of Saarbrücken and the Dr. rer. nat. degree from the university of Kiel. His research interests include query languages, graphical interfaces, and incomplete information.

Dirk Krämer is a member of the database group in the computing center of the university of Kiel. He received the Diploma in computer science from the university of Kiel. His research interests include visual query languages and distributed databases. 